\title{
Telescope polarization and image quality: Lyot coronagraph performance
}

\author{
J. B. Breckinridge ${ }^{1,2}$, and R. A. Chipman ${ }^{2}$ \\ 1. California Institute of Technology, Pasadena, CA. \\ 2. College of Optical Sciences, University of Arizona, Tucson, AZ
}

\begin{abstract}
In this paper we apply a vector representation of physical optics, sometimes called polarization aberration theory to study image formation in broadband white-light within astronomical telescopes and instruments. We describe image formation in-terms of interferometry and use the Fresnel polarization equations to show how light, upon propagation through an optical system become partially polarized. We make the observation that orthogonally polarized light does not interfere to form an intensity image. We show how the two polarization aberrations (diattenuation \& and retardance) distort the system PSF, decrease transmittance, and increase unwanted background above that predicted using scalar models. We show that in the presence of small geometric aberrations, polarization aberrations reduce coronagraph contrast and increase inner working angle (IWA) to limit the scientific yield of telescopes and instruments designed to detect \& characterize exoplanets.

The results we show here do not include a PSF for pointing and control, but are for the complex optical system only, and show the limit in the presence of zero pointing error and perfect alignment.

We apply the polarization aberration theory (PolAbT) described earlier (Breckinridge, Lam \& Chipman, 2015 ) to the fore-optics of the system designed for AFTA-WFIRST-CGI to obtain a performance estimate. Analysis of the open-literature design using PolAbT leads us to estimate that the WFIRST-CGI if built to the current design will give a contrast $(3 / D)$ in the $\sim 10^{-7}$ regime at the occulting mask. Not the $10^{-9}$ contrast
\end{abstract}

(3 /D) calculated by the project ${ }^{54}$. An accurate number for the contrast and inner working angle (IWA) awaits the opportunity to use POLARIS-M or other polarization code correctly, to map the complex wavefront through the end-to-end WFIRST CGI telescope-coronagraph system. These new values will change the exoplanet yield calculation to reduce the number of exoplanets WFIRST-CGI will observe.

We remind the reader: 1 . Polarizers are operators, not filters in the same sense as colored filters, 2. Adaptive optics does not correct polarization aberrations, 3. Calculations of both diattenuation and retardance are needed to model real-world telescope/coronagraph systems. 4 . The form birefringence of the highly reflecting mirrors needs to be determined.

Keywords: Polarization aberrations, exoplanet coronagraphs, form birefringence, Fresnel equations, PSF, complex wavefronts, image quality, optical thin films.

\section{INTRODUCTION}

An optical system corrected for geometric path difference errors is a necessary but not sufficient condition for the perfect image formation needed to directly image terrestrial exoplanets. Geometric (trigonometric) path difference errors are controlled using adaptive optics (tip-tilt \& wavefront), active metrology and precision pointing. However, image quality is also determined by several physical optics factors: diffraction, polarization, partial coherence, and chromatism all of which degrade image quality and are not corrected through the control of geometric path difference. The source of physical optics errors lies in the opto-mechanical packaging of optical elements, masks, stops and the thin film coatings needed to obtain high transmittance. Adaptive optics corrects wavefront errors described by geometric or optical path length errors but not those wavefront errors introduced by physical optics.

Breckinridge Kuper \& Shack ${ }^{1}$ first (1984) analyzed the use of the Lyot coronagraph to image distant exoplanets and modeled a system using the scalar approximation to the vector electromagnetic wave. They showed how scalarcomplex apodization of the exit pupil reveals an exoplanet in the presence of mirror fabrication errors. Earlier (1971) Breckinridge $^{2}$ showed that polarization internal to instruments contribute to spectrophotometric errors. Breckinridge \& Oppenheimer $^{3}$ (2004) showed that internal polarization plays an important role in exoplanet coronagraphy. Carson, et. al. ${ }^{4}$ provided a measurement of the polarization dependent PSF. Breckinridge $(2011)^{5}$ alerted the WFIRST-CGI science and technology development team to contrast degradation caused by internal polarization.

Space Telescopes and Instrumentation 2016: Optical, Infrared, and Millimeter Wave, edited by

Howard A. MacEwen, Giovanni G. Fazio, Makenzie Lystrup, Proc. of SPIE Vol. 9904,

99041C · (C) 2016 SPIE · CCC code: 0277-786X/16/\$18 - doi: 10.1117/12.2231242

Proc. of SPIE Vol. 9904 99041C-1 
Today, ground and space exoplanet coronagraphs are designed and built under the assumption that the scalar wave approximation to the vector electromagnetic wave $e^{6},{ }^{7}$, is adequate. Shaklan et. al. ${ }^{8}$ examined a terrestrial planet finder (TPF) coronagraph design using vector electromagnetic (E\&M) waves and concluded that for TPF designs vectorwaves were not necessary to develop a system to control scattered light to the level required at that time. Recently, Breckinridge $^{9}$ and Chipman ${ }^{10}$ used vector E\&M wave analysis with the polarization aberration tools developed by Chipman and others to model point-spread functions (PSF) for astronomical telescopes and discovered that several physical optics effects are in reality very important for the design of high performance coronagraphs.

In this paper we examine the role physical optics has in the direct imaging of exoplanets and suggest strategies to minimize the negative effects of aberrations introduced by physical optics. This text is divided into 5 sections. Section 1 is the introduction. Section 2 discusses image formation in the presence of polarization and reviews scalar wave image formation. In this section we show a dramatic example of what happens to the PSF in an extreme case of polarization variations across an exit pupil and provides a review of the Fresnel polarization equations. Section 3 describes vectorwave image formation using the Fresnel-Kirchoff diffraction integral and shows that, in the presence of polarization aberrations the PSF is the linear superposition of four PSF's. Section 4 summarizes polarization ray trace (PRT) and polarization aberration theory (PolAbT) and gives the detailed structure of the four PSF's: two have shape similar to an Airy diffraction pattern, but with a wider core and thus larger inner working angle, than that obtained by ignoring retardance of metal films. Two of the PSF's are displaced one relative to the other and the other two are fainter, but severely distorted and broader in extent. Conclusions from an analysis of a three-mirror bent Cassegrain are tabulated. In section 5 we apply our understanding of the physical optics properties of exoplanet coronagraphs to a discussion of how we will achieve maximum extinction at the focal plane by "impedance" matching the complex properties of the occulting mask to the E\&M content of the complex field at the focal plane. Section 6 provides a brief polarization aberration analysis of the AFTA-WFIRST-CGI system. And section 7 provides a brief discussion of our conclusions.

\section{TELESCOPE/INSTRUMENT SYSTEM IMAGE FORMATION}

\section{Scalar wave image formation}

In this section we provide a brief review of image formation modeled using scalar wave theory as a basis to extend the work into the more accurate vector representation.

Object space irradiance distribution can be decomposed into an ensemble of delta functions. The intensity or height of each delta function maps out the structure of the object. The optical system operates on the complex amplitude and phase associated with that intensity to form an image at the detector. Most astronomical sources in the visible region of the spectrum radiate broadband, incoherent thermal light.

The theory of image formation is developed using the schematic shown in Figure 1 below. The coordinate system we use in our analysis is shown in Fig 1 below. This system is in standard use by modern textbooks ${ }^{11},{ }^{12}$ on the physics of image formation. The object plane (\#1 in the system) is represented by Cartesian coordinates from the Latin alphabet $x_{1}, y_{1}$ the pupil plane (\#2 in the System) is represented by Cartesian coordinates from the Greek alphabet ${ }_{2}, 2_{2}$ and the image plane (\#3 in the System) is represented by Cartesian coordinates from the Latin alphabet $x_{3}, y_{3}$.

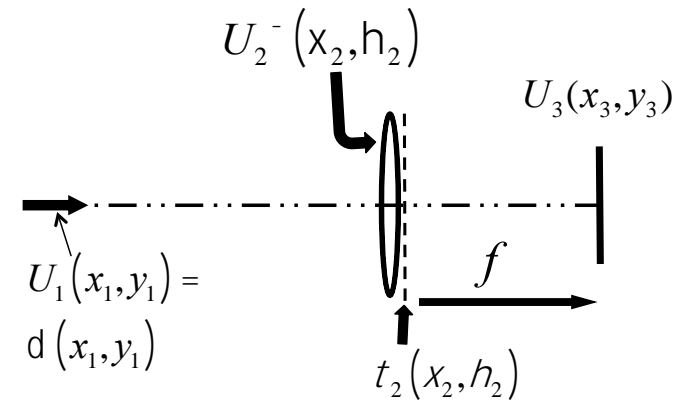

Figure 1 schematic of an optical system in the meridional plane. The object space complex field $U_{1}\left(x_{1}, y_{1}\right)$ is shown as a delta function $\left(x_{1}, y_{1}\right)$ to represent a star on-axis. This object space field is propagated by Fresnel diffraction to just to the left of the entrance pupil and is shown by $U_{2}\left({ }_{2},{ }_{2}\right)$. The pupil is shown to have a complex transmittance ${ }_{2}\left({ }_{2},{ }_{2}\right)$ at plane 2 . The optical system, shown 
here schematically has a lens of focal length $f$, and provides the optical power to create the field $U_{3}\left(x_{3}, y_{3}\right)$ at an image plane 3 .

The scalar complex amplitude and phase across the image plane is found by standing at the image plane (\#3) in Fig 1 and looking to the left, or back through the system toward the object. The Fresnel-Kirchoff diffraction integral is used to model the propagation of scalar electromagnetic waves through the optical system shown in Fig. 1. The complex amplitude and phase field $U_{3}\left(x_{3}, y_{3}\right)$ at the image plane is given by

$$
\begin{aligned}
& U_{3}\left(x_{3}, y_{3}\right)= \\
& \quad \int_{\infty}^{\infty} \int_{\infty}^{\infty}\left[U_{2}\left(\begin{array}{ll}
2 & 2
\end{array}\right)\right] \cdot{ }_{2}\left({ }_{2},{ }_{2}\right) \cdot \exp \left\{j \frac{2}{-}\left(x_{3} x_{2}+y_{3}{ }_{2}\right)\right\} d d
\end{aligned}
$$

Where $\mathrm{K}$ is a constant, the integral is taken over the complex field across the exit pupil, $U_{2}\left({ }_{2},{ }_{2}\right)$ of the optical system whose focal length is $f,{ }^{-}$is the quasimonochromatic wavelength of light. Eq 1 is written for the scalar wave solution to Maxwell's equations and the not vector wave solution to Maxwell's equation. The amplitude and phase complex properties across the exit pupil are contained in the scalar term,

$$
{ }_{2}\left({ }_{2},{ }_{2}\right)=A_{2}\left({ }_{2}, \quad 2\right) \exp \left\{i_{2}\left({ }_{2}, 2\right)\right\}
$$

Where $A_{2}\left({ }_{2}, 2\right)$ varies between 0 and 1 and describes amplitude part of the complex wave as a function of position across the exit pupil. The phase properties at each point across the exit pupil are described by ${ }_{2}\left({ }_{2},{ }_{2}\right)$.

To the left in Fig 1, we have a point source represented by a delta function. This point source is mapped onto the image plane. We record intensity at the image plane and define the image plane irradiance distribution for this point source to be:

$$
P S F_{\text {Scalar Diffraction }}\left|U_{3}\left(x_{3}, y_{3}\right)\right|^{2} \text {. }
$$

Next if we let the object space irradiance be represented by $I_{\text {Object }}\left(x_{1}, y_{1}\right)$ and the image space irradiance represented by $I_{\text {Image }}\left(x_{3}, y_{3}\right)$ and use the theoretical development of Goodman, we can write,

$$
I_{\text {Image }}\left(x_{3}, y_{3}\right)=I_{\text {Object }}\left(x_{1}, y_{1}\right) \quad P S F,
$$

Where the symbol denotes the convolution operator.

The analysis above reviews the classical scalar approach to modeling optical systems. Our new approach, needed for high contrast optical systems is to model white-light electromagnetic radiation as a vector. To understand the need for vector-wave physical optics, we need to review the source of polarized light within an optical system and understand the complex (amplitude and phase) wavefront at the focal plane $U_{3}\left(x_{3}, y_{3}\right)$ where coronagraphers place the occulting mask to control scattered light to one part in $10^{11}$. Correct design of the optimum occulting mask requires our new vector E\&M approach.

\section{Role of vector waves in image formation}

An experiment using linear orthogonal polarizers and a telescope shows the role of vector waves in image formation. Figure 2 shows the effects of adding polarizers to an optical system: Top left shows an open, unmasked exit 
pupil of a telescope with zero geometric wavefront error. Top right shows the shape of the PSF recorded with the pupil on the top left. Bottom left shows the same telescope pupil as that shown in the upper left, with two linear polarizers over the top, one aligned orthogonally to the other. Horizontally polarized light is admitted to the left-hand side of the pupil and vertically polarized light is admitted to the right-hand side of the pupil. The bottom right shows the PSF recorded using the pupil on the bottom left. Note that with no polarizer the angular resolution is not position-angle dependent, however, with the polarizer the angular resolution is position angle dependent. Astronomers define position angle as the rotation angle in the plane of the sky, or in this case the plane of object space. That is, the upper right in Figure 2 shows that the angular resolution is the same in all directions from the axis, whereas the lower right in Figure 2 the angular resolution is not the same in all directions from the system axis. Resolution in the horizontal direction exceeds that in the vertical direction.

\section{Figure 2 PSF's shown for a telescope with zero geometric wavefront aberration without (upper and with (lower) polarizers.}

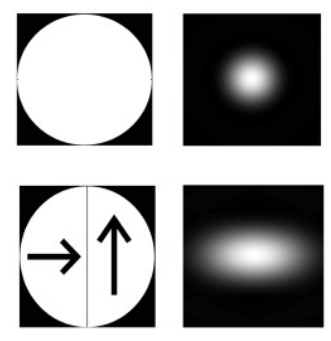

Orthogonally polarized white light does not interfere to create an image. In Fig 2, the lower left image of the exit pupil the polarized radiation from the left portion of the exit pupil does not interfere with the orthogonally polarized radiation from the right portion of the exit pupil. Therefore the PSF is elongated in the horizontal direction. In this case the PSF is the scalar sum (linear superposition) of two images of a " $\mathrm{D}$ " shaped aperture, not the vector sum across the circular aperture shown in the upper right panel in Fig 2. The inner working angle is larger in the horizontal direction than it is for the vertical direction. This means that a coronagraph mask positioned at the image plane that is designed using scalar theory and applied to a system with polarization aberrations (like WFIRST-CGI), would leak large amounts of light around the occulting mask to flood the coronagraph and block light from exoplanets to reduce exoplanet yield perhaps to the level of uselessness.

Although this is a rather dramatic example and no one would intentionally place orthogonal linear polarizers over their telescope pupil, this shows that any source of polarization change across the exit pupil will result in distortion of the PSF at some level and result in light leakage around the occulting masks now designed using scalar theory only.

In the next section we identify sources within the telescope/coronagraph optical system that polarize light. Current astronomical science measurement objectives requires high transmittance optical systems, which in turn require high reflectivity broad-band optical thin films. As the white-light electromagnetic wave propagates through the optical system it becomes partially polarized. The Fresnel polarization equations give the magnitude and sign of this polarization and are described in the following section.

\section{Fresnel polarization}

Here we examine the source of phase and amplitude changes within astronomical telescopes and instruments. Systems require mirrors coated with metals (e.g. Al or Ag) to give high surface reflectance and thus maximize system transmittance. These mirrors are overcoated with a dielectric material that serves two purposes: 1. A transparent mechanical barrier coat to inhibit oxidation and surface abrasion 2. Enhance the reflectivity at select wavelengths. In the next section we show that these metal surfaces partially polarize light. These dielectric overcoats contribute by either adding to or subtracting from the polarization aberrations caused by the metal mirror. Dielectric coatings add a degree of complexity that will affect coronagraph contrast and the inner working angle (IWA), and are not discussed here other than to mention they can be used to a limited extent to mitigate polarization aberrations over a narrow bandwidth in real optical systems.

In figure 2, above we saw that the change in polarization across the exit pupil affects image quality. Broadband unpolarized white-light is a characteristic of nearly all-astronomical sources and is divided equally into two orthogonally polarized beams for the derivation below. We represent unpolarized light by two orthogonal Eigenvector states and, for 
this example we select linearly polarized Eigenvector states. The Fresnel equations ${ }^{1314}$ are used to model the behavior of a vector electromagnetic complex wave interacting with a metal or dielectric surface (mirror).

A-J Fresnel in 1823 described the theory for interactions of electromagnetic radiation with dielectrics and metals. These relationships were developed further ${ }^{15}$ and are the basis of the commercial field of ellipsometry ${ }^{16}$. Here we describe those relationships.

Consider incoherent white-light incident at angle ${ }_{0}$, onto a metal mirror with isotropic properties. This metal mirror has a wavelength dependent complex index, $N_{1}(\quad)=n_{1}(\quad)+i k_{1}(\quad)$. The Eigenstates of reflection are the s (perpendicular) and $\mathrm{p}$ (parallel) polarized components. A portion of the beam reflects at the incidence angle ${ }_{0}$ (Snell's Law) and another portion (a damped evanescent wave) penetrates a short distance into the metal at the complex refraction angle of $\quad{ }_{1}$ given by Snell's law $^{17}$ and is absorbed to heat the metal. This complex angle is given by

$$
{ }_{1}=\operatorname{arcos}\left\{\left(\sqrt{N_{1}^{2} \quad N_{0}^{2} \sin { }_{0}^{2}}\right) / N_{1}\right\}
$$

The complex reflectivities for light in the $\mathrm{p}$ and s polarizations are given by ${ }^{33}$

$$
r_{p}=\frac{\tan \left({ }_{0}{ }_{1}\right)}{\tan \left({ }_{0}+{ }_{1}\right)} \text { and } r_{s}=\frac{\sin \left({ }_{0}{ }_{1}\right)}{\sin \left({ }_{0}+{ }_{1}\right)}
$$

Two polarization effects occur. 1. a phase shift and 2. a polarization-dependent absorption.

\section{Phase Shift}

The phase shift occurs between the waves associated with each of the two polarizations. We use the notation $\psi$ to express the angle of retardance of the s-polarized light relative to the p-polarized light. Equation 7 defines the polarization aberration,

$$
\tan =\tan \left(\begin{array}{cc}
s & p
\end{array}\right)=\left|r_{s}\right| /\left|r_{p}\right|
$$

Equation 7 gives us the retardance for a single ray propagating through the system. An image requires an array or an ensemble of rays. When we trace multiple rays from a single point in object space, the tangent of the retardance becomes

$$
\tan (,)=\tan \left({ }_{s}(,) \quad{ }_{p}(,)\right)=\left|r_{s}(,)\right| /\left|r_{p}(,)\right|
$$

Where $($, )are coordinates across the pupil. And we see that the retardance varies across the pupil.

\section{Polarization dependent absorption}

The reflectivity is polarization dependent, with the result that reflection acts as a partial polarizer. The term diattenuation is used to remind us that there are two (Di) measurements required here. The diattenuation, $D$ at each point $(, \quad)$ across the pupil is given by

$$
D(, \quad)=\frac{\left|r_{s}(,)\right|^{2}\left|r_{p}(,)\right|^{2}}{\left|r_{s}(,)\right|^{2}+\left|r_{p}(,)\right|^{2}}
$$

Where $r$ is the complex reflectivity for $s$ and $p$ light respectfully given in Eq 6 above. Metallic reflection acts as a weak polarizer, and $D$ varies from zero (nonpolarizing) to one for ideal polarizers. Astronomical optical systems require 
large étendu (area times solid angle), which requires large optics. However, the volume for spacecraft bus is required to be compact to fit inside launch shrouds. These two requirements conflict and often lead to many fold mirrors in the instrument which, unless designed properly will, in turn, lead to large internal polarization with the concomitant loss in transmission, caused primarily by diattenuation and loss in image quality, caused primarily by retardance.

Note there are two polarization aberrations: 1. Diattenuation is commonly used to model a polarization dependent reflectivity and 2 . Retardance is used to model a polarization dependent change in the phase of the complex wave upon reflection.

\section{IMAGE FORMATION IN THE PRESENCE OF POLARIZATION}

\section{Vector wave image formation}

In the previous section we examined the scalar diffraction equation 1, above. If the source is partially polarized and the telescope has a polarization dependent transmission, then Eq. 1 is written as:

$$
\begin{aligned}
& \vec{U}_{3}\left(x_{3}, y_{3}\right)= \\
& \mathrm{K} \int_{-\infty}^{\infty} \int_{-\infty}^{\infty}\left[\vec{U}_{2}^{-}\left(\xi_{2}, \eta_{2}\right) \vec{\tau}_{2}\left(\xi_{2}, \eta_{2}\right)\right] \cdot \exp \left\{-j \frac{2 \pi}{\bar{\lambda} f}\left(x_{3} \xi_{2}+y_{3} \eta_{2}\right)\right\} d \xi d \eta
\end{aligned}
$$

The complex wavefront for unpolarized, incoherent astronomical sources becomes partially polarized upon propagation through an astronomical telescope and instrument that contains metal mirrors and dielectric surfaces. A Jones matrix ${ }^{18,19}$ whose values change for different ray-paths across the exit pupil describes this wavefront. This spatially dependent matrix is called the Jones pupil ${ }^{18}$, we write this short hand for the complex field at each point $(, \quad)$ across the exit pupil:

$$
\left|\begin{array}{cc}
A_{X X} e^{i}{ }_{X X} & A_{X Y} e^{i}{ }_{X Y} \\
A_{Y X} e^{i_{Y X}} & A_{Y Y} e^{i_{Y Y}}
\end{array}\right|=\left|\begin{array}{cc}
J_{X X} & J_{Y X} \\
J_{X Y} & J_{Y Y}
\end{array}\right|
$$

On the LHS of Eq. 11, A is amplitude and is phase of the electric field for each of the 4 component waves in an arbitrarily selected X,Y Eigen basis-set at points , across the exit pupil. Subscript XX refers to the complex field exiting polarized in $X$ resulting from the incident field with $X$ polarization, as matrix multiplication would imply. A similar convention extends to the subscripts YY, YX and XY. Ideally, the Jones pupil would be the identity matrix for all ray paths and no undesired polarization change would occur. ${ }^{19}$ That is, the off-diagonal elements in the matrix shown in Eq.11 would be zero. During image formation with incoherent light, none of these four Jones pupil components form interference fringes with each other ${ }^{20,21}$. Each is diffracted separately by scalar diffraction theory to calculate the four components of the amplitude response matrix, which is the generalization of the amplitude response function of diffraction theory ${ }^{22}$.

The vector transmittance of the telescope $\vec{\tau}_{2}\left(\xi_{2}, \eta_{2}\right)$ is written,

$$
\vec{\tau}_{2}\left(\xi_{2}, \eta_{2}\right)=\left|\begin{array}{cc}
J_{X X} & J_{Y X} \\
J_{X Y} & J_{Y Y}
\end{array}\right|_{\xi, \eta}
$$


The telescope/coronagraph system complex transmittance across the exit pupil depends on the vector of the electromagnetic field at point , within the exit pupil. The complex electric field $u_{3}\left(x_{3}, y_{3}\right)$ at the image plane, for an on-axis unpolarized star of unit brightness follows from the Fresnel Kirchoff diffraction integral and is written:

$$
\begin{aligned}
& u_{3}\left(x_{3}, y_{3}\right)=
\end{aligned}
$$

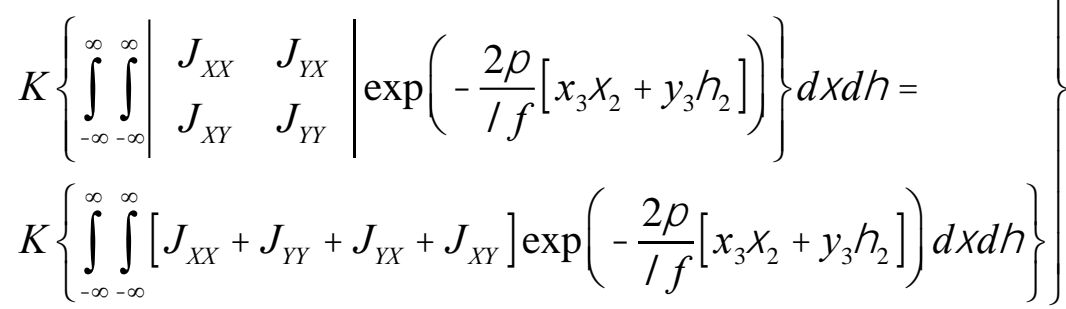

Where $K$ is a constant and we assume that the optical power of the system is not vector (polarization) dependent and we have multiplied out the matrix to emphasize that we will discover these 4 terms will be shown to be independent and not correlated. To calculate the intensity $I_{3}\left(x_{3}, y_{3}\right)$ that we will measure, we take

$$
I_{3}\left(x_{3}, y_{3}\right)=\left|u_{3}\left(x_{3}, y_{3}\right)\right|^{2} \text {. }
$$

And from Eq 13, we find the intensity to be

$$
\begin{aligned}
& I_{3}(x, y)= \\
& =\left|u_{3}(x, y)\right|^{2}=\mid K\left\{\int_{\infty}^{\infty} \int_{\infty}^{\infty}\left[J_{X X}+J_{Y Y}+J_{Y X}+J_{X Y}\right] \exp \left(\frac{2}{f}\left[\begin{array}{ll}
x_{3} 2 & +y_{3}
\end{array}\right]\right) d d\right\}
\end{aligned}
$$

Since we are observing a star, which is a thermal broadband white-light source, it is reasonable to assume that the complex electric fields given by $J_{X X}, J_{Y Y}, J_{Y X}$ and $J_{X Y}$ are statistically uncorrelated and therefore incoherent. The cross product terms within the modulus squared shown in Eq 15 are zero. Consequently, Eq. 15 can be expanded to gives,

$$
\begin{aligned}
& I_{3}\left(x_{3}, y_{3}\right)=
\end{aligned}
$$

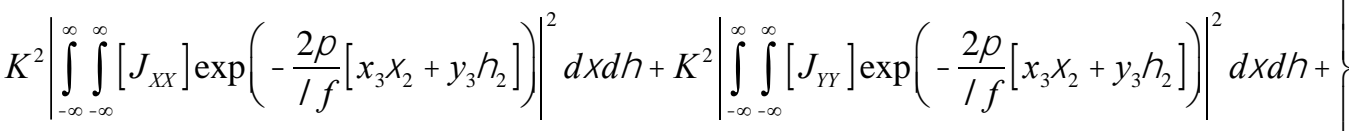

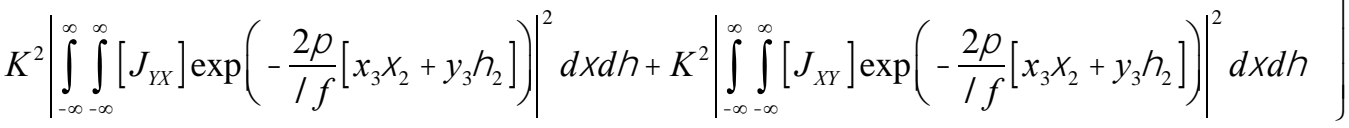

And we see that we have four point-spread functions, one each for the uncorrelated fields $J_{X X}, J_{Y Y}, J_{Y X}$ and $J_{X Y}$ and the image plane point spread function is the linear, incoherent superposition of four PSF's as shown in Eq. 17 below.

$$
I_{3}\left(x_{3}, y_{3}\right)=\left.\underline{I_{3}\left(x_{3}, y_{3}\right)}\right|_{X X}+\underline{\left.I_{3}\left(x_{3}, y_{3}\right)\right|_{Y Y}}+\underline{\left.I_{3}\left(x_{3}, y_{3}\right)\right|_{Y X}}+\underline{\left.I_{3}\left(x_{3}, y_{3}\right)\right|_{X Y}}
$$

The subscript $\mathrm{XX}$ means $\mathrm{X}$ light entering the system polarized in the $\mathrm{X}$ direction mapped into the $\mathrm{X}$ direction or $\mathrm{X}<=\mathrm{X}$, and similarly for light in the $\mathrm{Y}$ direction. The subscript $\mathrm{XY}$ refers to light entering the system polarized in the $\mathrm{Y}$ direction that exits the system in the $\mathrm{X}$ direction. Note that at the focal plane the terms $\left.\underline{I}_{3}\left(x_{3}, y_{3}\right)\right|_{X X}$ and 


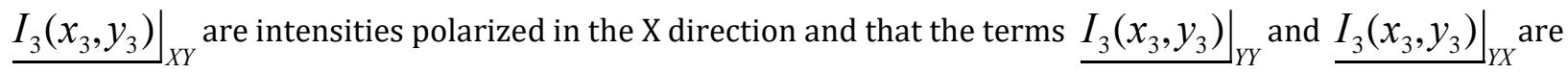
intensities polarized in the $\mathrm{Y}$ direction.

Figure 4 below shows these four point spread functions (PSF).

\section{POLARIZATION RAY TRACE (PRT) AND POLARIZATION ABERRATION THEORY (POLABT) Polarization ray trace (PRT)}

Polarization ray tracing (PRT) is a technique for calculating the polarization matrices for ray paths through optical systems $\mathrm{s}^{23,24,25,26,27,28}$. Polaris- $\mathrm{M}^{29}$ was built from the ground up to calculate polarization effects in optical systems. It is based on a $3 \times 3$ polarization ray tracing calculus ${ }^{46}$. Diffraction image formation of PolAb beams is then handled by vector extensions to diffraction theory ${ }^{30,31,32,33}$. A calculation of the polarization point spread matrix and optical transfer matrix can be seen in Section 4 of Ref. 42.

\section{Polarization aberration theory (PolAbT)}

Polarization aberration theory (PolAbT) describes the polarization effects of diattenuation, retardance, and apodization in a series expansion, where a cascade of terms separate mathematically the largest effects, from smaller effects and associate these polarization related image defects with constructional parameters and coating performance metrics. ${ }^{34,35}$ For example one term, retardance tilt, is strongly associated with fold mirrors and causes the XX and YY image components to shift with respect to each other, making the PSF slightly elliptical ${ }^{1}$. Another term retardancedefocus causes astigmatism from primary and secondary mirrors, which is polarization dependent; the orientation of the retardance rotates with the orientation of an incident linear polarization. ${ }^{36}$.

PRT generates very large files of numbers, at least eight times more than a conventional ray trace, leaving the designer and management a substantial data interpretation task of the aberrations represented in a higher dimensional polarization space. PolAbT is more difficult analytically than PRT, but it simplifies the ray tracing results into a small number of "terms" which are understood and addressed in an uncoupled manner. This enables us to manage polarization aberrations in more complicated systems, such as WFIRST-CGI.

A distinction between the two is seen in the comparison between classical geometric aberration ray trace (analogue to PRT) and the structural aberration coefficients ${ }^{37}$ (analogue to PolAbT) used by advanced designers to arrive quickly at near-optimized designs. Thus using PolAbT together with PRT is far more powerful than either method alone.

\section{Polarization ray-trace}

The output of a CAD ray-trace computer program is combined with Fourier optics to calculate point spread functions. Figure 3 shows a side view of a typical optical system with a fan of rays originating from a point on the object and passing through an optical system with $\mathrm{k}$ surfaces to the system exit pupil. Each ray strikes a real physical surface at a known angle of incidence (no paraxial approximation).

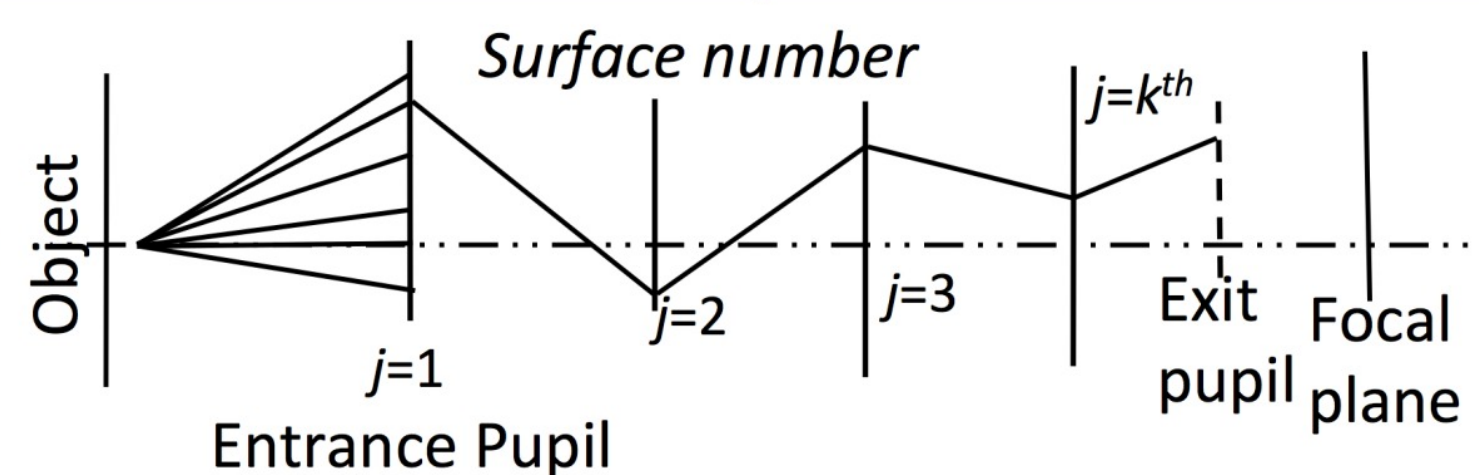

Figure $3 \mathrm{~A}$ fan of rays is shown passing from the object plane through an optical system with $k$ surfaces before the exit pupil. For descriptive purposes a fan of rays is shown traced to surface $j=1$ and then a general single ray continues on to the exit pupil at surface $j=k+1$. 
We know the physical properties of each surface. Each surface in an optical system is either a reflecting metal or a dielectric. Using the Fresnel equations, discussed in section 2 we calculate values for each of the four complex entries in Eq. 16, for each ray intercept through the system. We compute the multiplicative amplitude and cumulative phases for both perpendicular and parallel light and map these into four arrays of complex numbers across the exit pupil. We then take a digital FFT of each set of these 4 arrays of complex-field points to calculate the four PSF's.

\title{
Three-mirror imaging telescope polarization ray trace (PRT)
}

The PolAb and diffraction image formation of a 3-mirror telescope system (fig 3 ref. 1) classical Cassegrain were analyzed with the Polaris-M software to evaluate the order of magnitude of polarization effects, which would be expected in a telescope/coronagraph. Polarization artifacts were discovered with "ghost PSFs" about twice the size of the Airy disk at $10^{-5}$ of the peak intensity. This 3-mirror optical system is a much simpler optical system than the 18-mirror WFIRST-CGI. PRT for an 18-mirror system is very labor intensive and we developed our theory based on the much simpler 3-mirror system and derived the more general PolAbT and used that to predict performance of the 18 mirror WFIRST-CGI at the image plane location of the complex occulting mask.

For the three-mirror bent Cassegrain telescope, we adjusted the surface figures and vertex intervals so that geometric aberrations were zero for the on-axis PSF. All residual aberrations were therefore caused by polarization, not geometric aberrations. The primary mirror is $2.4 \mathrm{~m}$ dia. and F/\# 1.2, the curvature on the secondary was adjusted to give a system $F / \#=8$. The position of the secondary along the system axis was adjusted so the $F / \# 8$ converging beam reflected from a flat 45 -degree mirror to deviate the beam by 90 -degrees to a focal plane underneath the primary. Our calculations assumed bare $\mathrm{Al}$ at $=800 \mathrm{~nm}$, where the complex index of refraction, $N_{1}$ is $N_{1}=2.8+8.45 i$. Each surface contributes its own polarization for each ray that strikes it. The cumulative complex transmittance for each point in the exit pupil is found by multiplying the $3 \times 3$ polarization ray tracing matrices at each of the 3 surfaces ${ }^{38}$. This provides in $3 \mathrm{D}$ the exiting polarization state for arbitrary incident states. The Jones field at the exit pupil is Fourier transformed to find the EM field at the focal plane for all incident polarization states. The amplitude of the field contains four PSF components ${ }^{39}$, shown in Fig. 4, below.

This Cassegrain telescope is illuminated by a thermal white-light star on-axis. The image plane irradiance is found to be the linear superposition of the four amplitude PSF's ${ }^{40}$ shown in Fig 4. Not visible at this scale is the fact that the centroid of each of the four PSF's are shifted slightly, one with respect to the other, to destroy rotational symmetry about the axis.
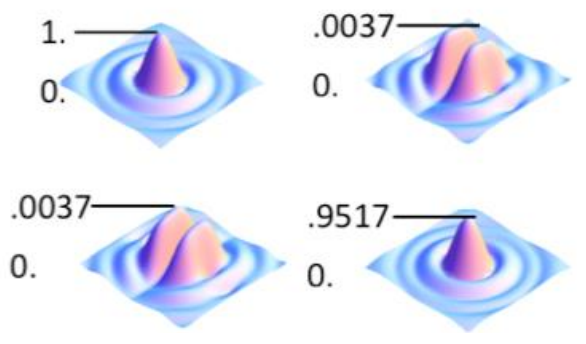

\begin{abstract}
Figure 4, a single star on axis in object space produces a four-part ARM given in Eq. 11. Here we plot the amplitude (A) for these complex elements of the $2 \times 2 \mathrm{ARM}$, as they appear nearly super-posed at the focal plane of the Cassegrain telescope. The scale or size of each of these ARM elements is wavelength dependent. We refer the two off diagonal highly structured PSF's as ghost PSF's.
\end{abstract}

The amplitudes presented in Fig 4 are given in Eq. 11 and show the terms within the amplitude response matrix. The point spread functions are calculated by taking the modulus squared of these terms. The shapes of the off-diagonal ghost PSFs, IXY and IYX are highly irregular and show a "lumpy" structure that might be confused with an exoplanet. The spatial extent of each of these off-diagonal ghost PSFs, IXY and IYX, are twice as large as the spatial extent of the on- 
diagonal images. The detailed analysis of these images shows several issues of concern for the WFIRST-CGI, which contains 15 additional mirrors (before the occulting mask) than we used for the three-mirror bent-Cassegrain for the calculation in Fig 4.

In Fig 4, the two principal images $\mathrm{I}_{\mathrm{XX}}$ and $\mathrm{I}_{\mathrm{YY}}$ are shifted by 0.625 mas with respect to each other due to a linear variation of retardance at the mirror - additional mirrors (like we have in CGI) in the path will increase this separation. Each of the principal images, $\mathrm{XX}$ and YY, is slightly astigmatic, but with opposite astigmatism sign (rotated $90^{\circ}$ ). The PolAb cause polarization crosstalk between $\mathrm{X}$ and $\mathrm{Y}$ polarized light, the off-diagonal elements, which although weak, 0.0037 in amplitude, $10^{-5}$ in flux, has a much larger extent than the Airy disks of the principal components - additional mirrors (like we have in CGI) will increase the flux in the "ghost PSF's" calculated from the off-diagonal elements shown in Fig. 4. The intensities of the off diagonal profiles shown in Figure 4 above increases as the square of the number of mirrors and are dependent on ray path incidence angle, as well as the value of the metal thin film form birefringence.

Primary and secondary mirror coatings introduce astigmatism on-axis ${ }^{41}$ which couples light into the orthogonal polarization state in Maltese cross type patterns, yielding the ghost PSFs. Focusing through fold mirrors introduces a linear variation of retardance, putting a different linear phase shift on the two principal components, shifting them in opposite directions! We derived eighteen scaling relations or design rules for these system parameters using PolAb theory ${ }^{9}$.

Fig 5 (top) below shows a plot of the $\log _{10}$ of the irradiance in the meridional plane at the image for $I_{X X}$ (the solid line) and $I_{Y X}$ (the dotted line) while Fig 5 (lower) is a "face-on" map of $I_{Y X}$ with the classic Airy diffraction rings superposed.

Note that the positions of the zeros in the PSF associated with $I_{X X}$ are not superposed on those associated with $I_{Y X}$. Therefore ghost images could be misinterpreted as a "false alarm" candidate exoplanet.
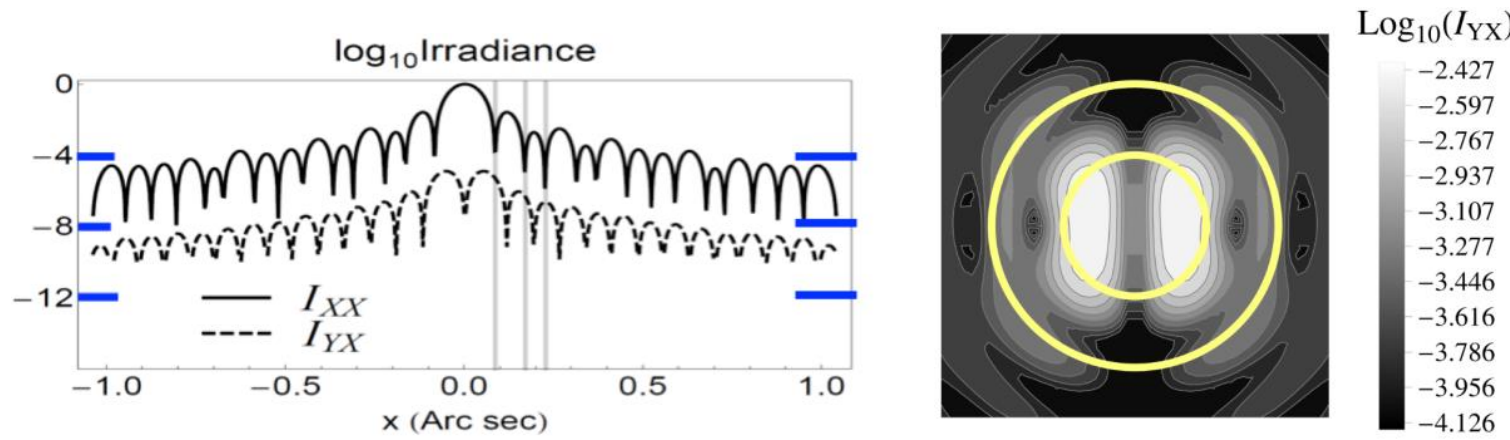

Figure 5 (upper) plots (solid line) of the $\log _{10}$ of the irradiance in the meridional plane at the image for the irradiance distribution. In the upper part of the figure, the lower dotted line shows the $\log _{10}$ irradiance for the irradiance distribution. The lower image in this figure is the "face-on" appearance of Iyx or the PSF Image of the off diagonal terms shown in Figs 4 and Eq. 12. $=800 \mathrm{nmD}=\mathbf{2 . 4} \mathbf{~ m}$.

\section{Polarization changes across the PSF}

To first order the optical system of a coronagraph is isoplanatic and the fore-optics PSF for the star is the same as that for the planet. The image plane mask suppresses the field from the star out to a distance of $\sim 3 / D$. In the case of the 2.4 $\mathrm{m}$ Cassegrain system at $=800 \mathrm{~nm}$ where $3 \quad / D \quad 0.2$ asec we find that for both the star and its displaced planet the radius $r$ of the $90 \%$ encircled energy for $r_{X X}=r_{Y Y}=0.15$ asec and $r_{X Y}=r_{Y X}=0.36$ asec. Radiation from the cross-product terms extends into a 0.2 -asec coronagraph dark hole to create an irregular background pattern that will confuse exoplanet measurements.

\section{Conclusions from the analysis of the 3 mirror bent Cassegrain}

Breckinridge, Lam and Chipman (2015) ${ }^{42}$ and Chipman, Lam and Breckinridge (2015) ${ }^{43}$ used the POLARIS-M software to provide a detailed complex vector polarization analysis of a typical astronomical bent Cassegrain telescope 
comprised of an F/\# 1.2 primary and a secondary mirror whose optical power is sufficient to give an $\mathrm{F} / \# 8$ beam converging past a 45-degree flat mirror to a focal plane. The curvatures on the primary and secondary were designed to give zero geometric aberrations on axis. Coatings were assumed to be bare Al and wavelength 800nm. Several important facts about image quality in astronomical telescopes were found ${ }^{9,10}$ to affect high-contrast exoplanet coronagraphy and astrometry. Let = the ratio of the peak of on-diagonal PSF's shown in Fig 4 to the off-diagonal PSF's shown in the same figure. That is, is the ratio of the terms in the amplitude response matrix: $J_{X Y}$ or $J_{Y X}$ to the peak of $J_{X X}$ or $J_{Y Y}$ respectively. We use the astronomers' convention that the higher the contrast, the worse the system is for planet characterization. The lowest contrast possible is the most desirable.

1. The image plane PSF is the incoherent sum of 4 point nearly super-posed spread functions: two normalized near 1, but distorted PSF's and two highly distorted "Ghost PSF's", with long "tails", but a factor, fainter at the peak by approximately $4 \times 10^{-3}$ in amplitude as shown in Fig 4 above.

2. The magnitude of this factor depends on the wavelength and angles of incidence and the sign of the rays on each mirror in the path as well as the physical properties, of the highly reflective coatings on the mirrors. Increasing results in reduced contrast.

3. The number of fold mirrors in the optical system determines the factor which increases as the square of the number of "aligned" fold mirrors. Crossed fold mirrors ( $s-p$ at first becomes p-s at the next) at the same angle of incidence can compensate polarization. "Aligned" here means the number of mirrors which share their s-p orientation minus the number with the opposite p-s orientation. This is a possible method for reducing the magnitude of the cross-product terms in the ARM and desensitizing the system to the retardance polarization aberration.

4. The radius of the spatial extent of the $90 \%$ encircled energy of these two ghost PSF images is approximately twice as large as the mean radius of the two primary patterns: $J_{X X}$ and $J_{Y Y}$.

5. The PSF images for two orthogonal linearly polarization components of the ARM ( $J_{X X}$ and $\left.J_{Y Y}\right)$ are shifted with respect to each other, causing the PSF image for un-polarized point sources to become slightly elongated (elliptical) with a centroid separation on the order of 0.6 mas. The result is that the inner working angle does not have rotational symmetry; it is position angle dependent on the sky, which degrades the angular resolution of the system.

6. The full-width half-maximum of the $J_{X X}$ and $J_{Y Y}$ is larger than that for the classic Airy pattern calculated using scalar wave theory. This fact increases the inner working angle to degrade the angular resolution of the system and reduce exoplanet science yield.

\section{Applications}

At least two astronomical science measurement objectives are affected by internal polarization. These are: 1 Exoplanet characterization using coronagraphy and 2 Astrometry. The astrometric implications are discussed by Safonov ${ }^{44}$ and will not be repeated here. Rather we will concentrate on applications to characterize exoplanets and discuss this topic in more detail in the next section.

\section{LYOT CORONAGRAPH TO CHARACTERIZE EXOPLANETS}

\section{Optical schematic}

Figure 6 shows the optical path for the complex amplitude and phase as it passes through a typical exoplanet Lyot coronagraph. Several physical optics effects modify this complex wave before it reaches the detector. These are caused by the interaction of light and matter: polarization induced by mirrors, windows \& stops and diffraction produced by masks and stops and chromatic aberration which results from the wavelength-dependent indices of refraction of materials required to reflect (metal mirrors) and transmit (dielectrics, filters, prisms, etc.) light. 


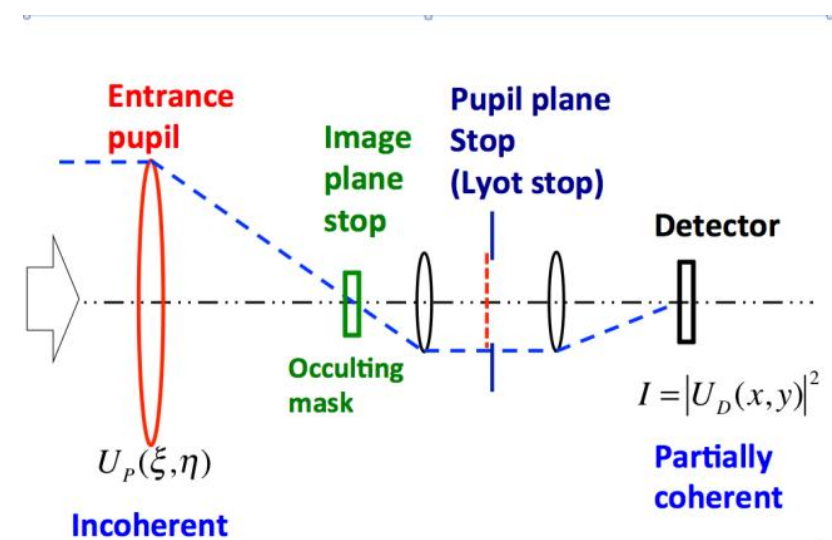

Figure 6. Optical schematic for a typical Lyot coronagraph. The Star/planet complex electromagnetic field enters the Lyot coronagraph system from the left and is focused onto a complex occulting mask located at an image plane stop. This image plane stop is at the front focus of a collimator lens. The optical power on the collimator is such that an image of the entrance pupil field is formed on the Lyot stop. The field is then focused on to the detector. Additional optics (not shown), in particular adaptive optics are inserted before the occulting mask to create a dark hole and the complex wavefront reflects from the several mirrors needed to package the system for flight. By the time light reaches the detector it is partially coherent.

The three-dimensional electric field from exoplanets is thermal white-light broadband, either reflected from the planetary system's parent star or emission from the planet or a mixture. The electric fields associated with the star and the planet are both spatially and spectrally incoherent ${ }^{45},{ }^{46},{ }^{47}$ at the source. This radiation travels through space, enters the telescope/coronagraph system and reflects from several mirrors to strike the occulting-mask stop at an image plane. The field is partially coherent at this stop. The stop must block by absorption or reflection almost all of the incident electric field from the star while passing as much as possible of the exoplanet field. Starlight diffracts around the entrance pupil and this electric field is scattered into the coronagraph to be blocked by the Lyot stop shown in Fig 6. The detector at the focal plane samples the modulus squared of the electromagnetic complex field, which appears as a speckle pattern caused by partial coherence of the wave-fields. This intensity speckle pattern is then digitized at an optimum dynamic range (number of bits) to obtain a high enough SNR for exoplanet characterization.

This intensity distribution contains information about the characteristics of the exoplanet as viewed through the "filter" of the telescope/coronagraph complex-vector transfer function (CVTF). This function, represented by the amplitude response matrix (ARM) ${ }^{48}$ varies significantly with the physical optics and the opto-mechanical design implementation. It is currently unknown for space or ground exoplanet coronagraph optical systems.

\section{Maximum extinction}

To minimize scattered light, the complex vector field at the image plane where the occulting mask is located (plane 3 in Fig. 6) must match the complex vector filter of the occulting mask. We use the formalism developed for optical processing of images ${ }^{49}$. From Eq. 17 we find the expression for the incoherent superposition of the four complex fields representing each of the 4 incoherent elements of the ARM to be:

$$
\vec{u}^{-}\left(x_{3}, y_{3}\right)=\mathcal{F}\left\{J_{X X}\right\}+\mathcal{F}\left\{J_{X Y}\right\}+\mathcal{F}\left\{J_{Y Y}\right\}+\mathcal{F}\left\{J_{Y X}\right\},
$$

Where $\mathcal{F}$ is the Fourier transform operator, each of the four terms is complex and the superscript - sign on $\vec{u}$ refers to the complex electric field infinitesimally ( ) to the left (-) or in front of the of the image plane. Note that it has been shown $\underline{\underline{10}}$ mathematically, confirmed observationally ${ }^{50}$ and relationships verified ${ }^{51}$ that the centroids of the terms in Eq. 18 are displaced slightly one from the other.

Let the transmittance of the image-plane occulting-mask be represented by the complex transmittance,

$\vec{\tau}\left(x_{3}, y_{3}\right)$, then the electric field infinitesimally ( ) to the right (+) of the complex occulting mask at the image plane is given by

$$
u^{+}\left(x_{3}, y_{3}\right)=u^{-}\left(x_{3}, y_{3}\right) \cdot \vec{\tau}\left(x_{3}, y_{3}\right)
$$


To achieve the contrast levels needed for terrestrial exoplanet spectrometry we need to minimize $u^{+}\left(x_{3}, y_{3}\right)$, over the wavelength bands of interest while maximizing the transmittance at the position of the exoplanet in orbit around the star.

To further control the unwanted radiation, this process needs to be repeated at the exit pupil to create the optimum complex EM Lyot stop. We need to satisfy the joint condition. This is a form of EM field impedance matching both at the image-plane occulting-mask and at the exit pupil Lyot stop under the conditions to maximize the field of the exoplanet at the entrance aperture to the spectrometer and to minimize the field from the star.

\section{PRELIMINARY POLARIZATION-ANALYSIS OF WFIRST-CGI $\underline{\underline{52}}$}

The detailed optical prescription for the WFIRST-CGI optical system was not made available for a polarization raytrace. However our analysis provided here can be used to provide insight into the polarization and image forming properties of this instrument in terms of estimates of the diattenuation and coronagraph contrasts that might be achieved.

Mirrors 1 and 2 are shared with the WFIRST-WFI. Mirrors 3 and 4 fold the light to the Tertiary followed by 13 additional mirrors, two of them separated deformable mirrors before the coronagraph image plane mask, for a total of 18 mirrors between the exoplanet and the image plane that contains the complex occulting mask. Each of these 18 mirrors is tilted to its ray propagation path and each member of the ray fan will become partially polarized in both diattenuation and retardance upon reflection. The exo-planet science path signal passes around the occulting mask to three more mirrors before the pupil mask, and then follows 9 more tilted mirrors to the detector. The science path from the primary mirror to the detector appears to have a total of 33 reflections, at incidence angles between $15^{\circ}$ and $20^{\circ}$. For the most part the optical system appears to be co-planar and diattenuation aberrations add.

Consider system transmittance in the s-polarized direction. Eq. 6 provides the reflectivity $r_{s}$ for a single mirror. We use equation 20 to calculate the total transmittance for the s-polarized ray. The total transmittance $T_{s}$ is calculated by taking the product of all of the surface (metal and dielectric) reflectance's in the optical ray path for the $s$-polarized ray to obtain:

$$
T_{S}=\prod_{j=1}^{j=k}\left[\frac{\sin \left({ }_{i}{ }_{E}\right)}{\sin \left({ }_{i}+{ }_{E}\right)}\right]_{j}
$$

Where the total number of physical surfaces is $k$ and $\quad{ }_{i}$ is the angle of incidence of the ray on the jth surface and ${ }_{E}$ is the angle of refraction penetrating into the metal at the jth surface by the evanescent wave. The angle ${ }_{E}$ is calculated using Eq. 5 and the complex index of the metal. The angle ${ }_{i}$ is determined by a computer aided design program such as Code V or Zemax and is the ray intercept angle on the physical surface of the mirror. We use equation 21 to calculate the total transmittance for the p-polarized ray. The total transmittance $T_{p}$ is calculated by taking the product of all metal and dielectric surface reflectances in the optical ray path for the $p$-polarized ray to obtain,

$$
T_{P}=\prod_{j=1}^{j=k}\left[\frac{\tan \left(\begin{array}{ll}
{ }_{i} & \\
E
\end{array}\right)}{\tan \left({ }_{i}+{ }_{E}\right)}\right]_{j}
$$

Where the total number of physical surfaces is $k$ and $\quad{ }_{i}$ is the angle of incidence of the ray on the $j$ th surface and ${ }_{E}$ is the angle of refraction penetrating into the metal at the $j$ th surface by the evanescent wave. The angle ${ }_{E}$ is calculated using Eq. 5 and the complex index, $N_{i}$ of the metal.

It can be shown that the diattenuation $\mathrm{D}$ is given by

$$
D=\frac{T_{s} T_{p}}{T_{s}+T_{p}} .
$$

\section{Internal linear polarization effects on CGI}

At the detector, after a total of 33 reflections, the end-to-end telescope-coronagraph transmittance can be no higher than $\sim 1.5 \%$, depending on absorption at the star-occulting mask. For 33 bare aluminum mirrors at $15^{\circ}$ angle of incidence, an unpolarized star becomes ${ }^{52}$ about $15 \%$ polarized with the weakest image in light polarized parallel to the plane of incidence. The requirement ${ }^{53}$ to observe in unpolarized light cannot be met with the system as designed. 


\section{Retardance and diattenuation reduce performance}

Detailed optical prescriptions for neither the fore-optics, nor for the three coronagraphs proposed for WFIRST-CGI were available. All three of these coronagraph designs use the same 18 mirror-surface fore-optics system ${ }^{7}$. We are able to estimate contrast at the image-plane occulting-mask at the entrance to the coronagraph by applying the design rules given in Ref 9 in Eq 4 on page 449 or Eq. 6 on page 450, which were derived by applying PolAbT to the three-mirror bent Cassegrain.

To forecast contrast at the occulting mask we 1. Scaled these results and applied the Fresnel equations for both retardance and diattenuation to the 18 mirror-surface fore-optics system, assuming an average angle of incidence of 15 degrees. 2. Compensated for the fact that, without knowledge of the coatings, the values for our bare $\mathrm{Al}$ is different than for dielectric overcoated mirrors. 3. Recognized that the diattenuation and retardance are quadratic in the angle of incidence and therefore one mirror at $45^{\circ}$ is approximately equal to 18 mirrors at $\sim 15^{\circ}$. We estimate contrast to be $\sim 10^{-7}$ or higher at $3 / D$.

\section{CONCLUSION}

The published performance of the WFIRST-CGI appears to have been modeled using only diattenuation polarization aberrations and did not include the retardance polarization aberration. The published contrast ${ }^{54}$ is contrast is $\sim 10^{-9}$ at $3 / D$, see ref. \# 54 Table 8 , page 47 . We estimate that the calculated contrast including all of the polarization terms is 2 to 3 orders of magnitude larger (poorer performance) than the value published ${ }^{54}$.

The form birefringence of the highly reflecting mirrors is largely unknown and has been of little importance until now when need to build telescope/coronagraph system at the 10-10 contrast level for the HabEx mission needs to be determined. Polarization mitigation technologies are needed for AFTA-WFIRST-CGI to increase its threshold raw contrast and increase the system scientific yield to increase the success of the mission.

\section{REFERENCES}

[1] Breckinridge, J. B., T. G. Kuper and R. V. Shack (1984) "Space telescope low scattered light camera: a model," Optical Engineering, 23, 816-820. also Proc. SPIE 331, 395-403, 1982.

[2] J. B. Breckinridge (1971) "Polarization Properties of a Grating Spectrograph," Applied Optics, 10, 286-294. [3] J. B. Breckinridge and B. Oppenheimer (2004), "Polarization Effects in Reflecting Coronagraphs for White Light Applications in Astronomy," ApJ, 600, pp. 1091 - 1098.

${ }^{4}$ Joseph Carson, James Breckinridge, John Trauger... (2006) "The effects of instrumental elliptical polarization stellar point spread function fine structure" Proc. of the SPIE 6265-3M.

5 WFIRST-CGI science and technology definition team (STDT) 2 $2^{\text {nd }}$ meeting Spring 2011 GSFC

${ }^{6}$ N. Zimmerman, A. J. E. Riggs, N. J. Kasdin, et. al. (2016) "Shaped pupil coronagraphs: high contrast solutions for restricted focal planes", JATIS 2, 011012

${ }^{7}$ Hong Tang, M. Rud, R. Demers, R. Goullioud, et. al. (2015) “The WFIRST/AFTA Coronagraph Instrument Optical Design", Proc. SPIE 9605960504

${ }^{8}$ K. Balasubramanian, S. Shaklan, A. Give'on, E. Cady and L. Marchen (2011) "Deep UV to NIR space telescopes and exoplanet coronagraphs: a trade study on throughput, polarization, mirror coating options and requirements" Proc. SPIE $815151511 \mathrm{G}$

${ }^{9}$ Breckinridge, J., W.S.T. Lam and R. A. Chipman (2015) "Polarization aberrations in astronomical telescopes" Publ. Astron Soc. Of the Pacific 127:445-468

${ }^{10}$ R. A. Chipman, Wai Sze T. Lam and J. Breckinridge (2015) "Polarization aberrations in astronomical telescopes" Proc SPIE 9613 doi:10.1117/12.2188921

11 Joseph W. Goodman (2005) "Fourier Optics" Roberts and Company book ISBN 9747077-2-4

12 J. D. Gaskill (1978) "Linear Systems, Fourier Transforms and Optics", John Wiley \& Sons, NY 400 pages.

${ }^{13}$ Born, M. \& E. Wolf (1993) "Principles of optics", Pergamon Press- Fresnel Equations are derived on pp 627-633

${ }^{14}$ L. Ward (1988) "Optical Constants of Bulk Materials and Films", Adam Hilger, Bristol and Philadelphia, 245 page book.

${ }^{15}$ A. Thelen, (2002) "Design of Optical Interference Coatings" (McGraw-Hill, New York, 1988). AND 
H. A. Macleod, "Thin-Film Optical Filters" Institute of Physics, pp. 185-186. AND J.A. Dobrowolski, "Coatings and Filters," in Handbook of Optics, W.G. Driscoll and W. Vaughan, ed.(1978) (McGraw-Hill, New York), 8.1-8.124. ${ }^{16}$ R. M. A. Azzam and N. M. Bashara (2003) "Ellipsometry and Polarized Light", North Holland - Elsevier ISBN 0 $4444870164 ; 527$ pages.

${ }^{17}$ Ward, L. 1988, "Optical Constants of Bulk Materials and Films", Adam Hilger, p245

${ }^{18}$ R. C. Jones, (1941) "A New Calculus for the Treatment of Optical Systems: I. Description and Discussion of the Calculus", JOSA, 31, 488

${ }^{19}$ R. C. Jones, (1941) "A New Calculus for the Treatment of Optical Systems: 2. Application, JOSA, 31, 495.

${ }^{20}$ E. Wolf (2007) "Theory of Coherence and Polarization of Light", Chapter 8, Cambridge University Press, 225 page book. And J. W. Goodman (2015) "Statistical Optics", Wiley And J. D. Strong (1958) "Classical Optics", Freeman, page 178 interference of polarized light,.

${ }^{21}$ E. Wolf (2007) "Theory of Coherence and Polarization of Light", Chapter 8, Cambridge University Press, 225 page book. And J. W. Goodman (2015) Statistical Optics, Wiley And J. D. Strong (1958) Classical Optics, Freeman, page 178 interference of polarized light,.

${ }^{22}$ J.P. McGuire, Jr., R.A. Chipman (1990) "Diffraction image formation in optical systems with polarization aberrations I: Formulation and example", Journal of the Optical Society of America A., vol. 7, no. 9, pp. 1614-1626 (Sept. 1990).

${ }^{23}$ Bruegge, T. J. 1989, Proc. SPIE, 1166, 165

${ }^{24}$ Chipman, R. A. 1989a, "Polarization analysis of optical systems", Opt. Eng., 28, 90 and 1989b, Proc. SPIE, 1166, 79.

${ }^{25}$ Wolff, L. B. and D. J. Kurlander, "1990, IEEE Computer Graphics and applications" vol. 10, 6, 44-55.

${ }^{26}$ J.D. Trolinger, Jr., R.A. Chipman, D.K. Wilson, (1991) "Polarization ray tracing in birefringent media", Optical Engineering, vol. 30, no. 4, pp. 461-466.

${ }^{27}$ Yun, G., Crabtree, K. \& Chipman, R., 2011a, Appl. Opt. 50, 2855 \& 2011b, Appl. Opt. 50, 2866

${ }^{28}$ S.C. McClain, L.W. Hillman, R.A. Chipman, (1993) "Polarization ray tracing in anisotropic optically active media I, algorithms, \& II, theory and physics", Applied Optics vol. 10, no. 11, pp. 2371-2393.

${ }^{29}$ R. A. Chipman and W. S. T. Lam (2015) "The Polaris-M ray tracing program", Proc. SPIE 9613-18

${ }^{30}$ Kuboda, H. \& Inoué, S. 1959, JOSA, 49, 191

${ }^{31}$ Urbanczyk, W. 1986, Opt. Acta., 33, 53

${ }^{32}$ McGuire, J. P. \& Chipman, R. A. 1990, JOSA A, 7, 1614 \& 1991, JOSA A, 8, 6

${ }^{33}$ Tu, Y., Wang, X., Li, S. \& Cao, Y. 2012, Opt. Lett., 37, 2061

${ }^{34}$ R.A. Chipman, L.J. Chipman, (1989) "Polarization aberration diagrams, Optical Engineering", vol. 28, no. 2, pp. 100-106.

${ }^{35}$ J.P. McGuire, Jr., R.A. Chipman, (1994) "Polarization aberrations I: Rotationally symmetric optical systems and Polarization aberrations II: Tilted and decentered optical systems", Applied Optics, vol. 33, no. 2, pp. 5080-5107.

${ }^{36}$ D.J. Reiley, R.A. Chipman, (1994) "Coating induced wave front aberrations: On axis astigmatism and chromatic aberration in all reflecting systems", Applied Optics, vol. 33, no. 10, pp. 2002-2012.

${ }^{37}$ I. C. Gardner (1927) “Application of the Algebraic Aberration Equations to Optical Design", NBS Scientific Papers Vol 22 \# 550.

${ }^{38}$ G. Yun, K. Crabtree, and R. Chipman (2011) "Three-dimensional polarization ray-tracing calculus I: definition and diattenuation", Appl. Opt. 50, 2855-2865. AND

G. Yun, S. McClain, and R. Chipman (2011) “Three-dimensional polarization ray-tracing calculus II: retardance”, Appl. Opt. 50, 2866-2874.

${ }^{39}$ Figure 7: Breckinridge, J. , W.S.T. Lam and R. A. Chipman (2015) "Polarization aberrations in astronomical telescopes", Publ. Astron Soc. Of the Pacific 127:445-468

${ }^{40}$ Figure 7: Breckinridge, J. , W.S.T. Lam and R. A. Chipman (2015) "Polarization aberrations in astronomical telescopes" Publ. Astron Soc. Of the Pacific 127:445-468

${ }^{41}$ D.J. Reiley, R.A. Chipman (1994) "Coating induced wave front aberrations: On axis astigmatism and chromatic aberration in all reflecting systems", Applied Optics, vol. 33, no. 10, pp. 2002-2012

${ }^{42}$ J. Breckinridge, Wai Sze T. Lam and R. A. Chipman (2015) "Polarization Aberrations in Astronomical Telescopes: The Point Spread Function", Publications of the Astronomical Society of the Pacific (PASP), 127:445468. 
${ }^{43}$ R. A. Chipman, Wai Sze T. Lam and J. Breckinridge (2015) "Polarization aberrations in astronomical telescopes" Proc SPIE 9613 doi:10.1117/12.2188921

${ }^{44}$ B. Safonov (2015) On-sky demonstration of optical polar-astrometry, MNRAS 451, 3161-3172.

${ }^{45}$ M. Born and E. Wolf (1999) "Principles of Optics", Chapter 10: Partially coherent light, Cambridge University Press

${ }^{46}$ L. Mandel \& E. Wolf (1965) "Coherence Properties of Optical Fields", Rev. Mod. Phys 37, 231-280

${ }^{47}$ J. Goodman (2015) "Statistical Optics" Ch 5: Coherence of Optical Waves, Wiley and Sons publisher

${ }^{48}$ Breckinridge, Lam and Chipman (2015) "Polarization Aberrations in Astronomical Telescopes: The Point

Spread Function", Publications of the Astronomical Society of the Pacific (PASP), 127:445-468. Equation 8

${ }^{49}$ Joseph Goodman (2005) "Fourier Optics" Chapter on optical matched filters, Roberts and Company book ISBN

9747077-2-4

${ }^{50}$ B. Safanov (2015) “On-sky demonstration of optical polar-astrometry", NMRAS, 451, 3161-3172

${ }^{51}$ B. Safanov (2016) private communication

${ }^{52}$ Breckinridge, J. B., W.S.T. Lam and R. A. Chipman (2015) Polarization aberrations in astronomical telescopes, Publ. Astron Soc. Of the Pacific 127:445-468 - pp 456

${ }^{53}$ WFIRST-AFTA 2015 SDT report Table F-2 lists the requirement to observe in unpolarized light in filter bands 3,6, \& 7.

${ }^{54}$ Krist, J. \& B. Nemati \&B. Mennesson (2016) "Numerical modeling of the proposed WFIRST-AFTA coronagraphs and their predicted performances", JATIS 2, 011003 (jan-Mar 2016) pages 1 through 26. 